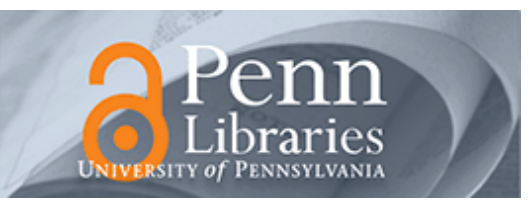

University of Pennsylvania

ScholarlyCommons

\title{
$6-2012$
}

\section{Multi-agent Flocking With Random Communication Radius}

\author{
Samuel Martin \\ Université de Grenoble \\ Arastoo Fazeli \\ University of Pennsylvania \\ Ali Jadbabaie \\ University of Pennsylvania, jadbabai@seas.upenn.edu \\ Antoine Girard \\ Université de Grenoble
}

Follow this and additional works at: https://repository.upenn.edu/ese_papers

Part of the Algebra Commons, Controls and Control Theory Commons, Dynamic Systems Commons, Other Applied Mathematics Commons, and the Probability Commons

\section{Recommended Citation}

Samuel Martin, Arastoo Fazeli, Ali Jadbabaie, and Antoine Girard, "Multi-agent Flocking With Random Communication Radius", . June 2012.

Martin, S., Fazeli, A., Jadbabaie, A., \& Girard, A. (2012). Multi-agent flocking with random communication radius. American Control Conference (ACC), pp.3871-3876. http://ieeexplore.iee.org/stamp/ stamp.jsp?tp=\&arnumber=6315594\&isnumber=6314593 (c) 2012 IEEE. Personal use of this material is permitted. Permission from IEEE must be obtained for all other uses, in any current or future media, including reprinting/republishing this material for advertising or promotional purposes, creating new collective works, for resale or redistribution to servers or lists, or reuse of any copyrighted component of this work in other works.

This paper is posted at ScholarlyCommons. https://repository.upenn.edu/ese_papers/616

For more information, please contact repository@pobox.upenn.edu. 


\title{
Multi-agent Flocking With Random Communication Radius
}

\author{
Abstract \\ In this paper, we consider a multi-agent system consisting of mobile agents with second-order dynamics. \\ The communication network is determined by a metric rule based on a random interaction range. The \\ goal of this paper is to determine a bound on the probability that the agents asymptotically agree on a \\ common velocity (i.e. a flocking behavior is achieved). This bound should depend on practical conditions \\ (on the initial positions and velocities of agents) only. For this purpose, we exhibit an i.i.d. process \\ bounding the original system's dynamics. We build upon previous work on multi-agent systems with \\ switching communication networks. Though conservative, our approach provide conditions that can be \\ verified a priori. \\ Keywords \\ Consensus, Stochastic Process, Random Networks, Graph Theory \\ Disciplines \\ Algebra | Controls and Control Theory | Dynamic Systems | Other Applied Mathematics | Probability \\ Comments \\ Martin, S., Fazeli, A., Jadbabaie, A., \& Girard, A. (2012). Multi-agent flocking with random communication \\ radius. American Control Conference (ACC), pp.3871-3876. http://ieeexplore.iee.org/stamp/ \\ stamp.jsp?tp=\&arnumber $=6315594 \&$ isnumber $=6314593$ \\ (C) 2012 IEEE. Personal use of this material is permitted. Permission from IEEE must be obtained for all \\ other uses, in any current or future media, including reprinting/republishing this material for advertising or \\ promotional purposes, creating new collective works, for resale or redistribution to servers or lists, or \\ reuse of any copyrighted component of this work in other works.
}




\title{
Multi-agent Flocking with Random Communication Radius
}

\author{
Samuel Martin, Arastoo Fazeli, Ali Jadbabaie and Antoine Girard
}

\begin{abstract}
In this paper, we consider a multi-agent system consisting of mobile agents with second-order dynamics. The communication network is determined by a metric rule based on a random interaction range. The goal of this paper is to determine a bound on the probability that the agents asymptotically agree on a common velocity (i.e. a flocking behavior is achieved). This bound should depend on practical conditions (on the initial positions and velocities of agents) only. For this purpose, we exhibit an i.i.d. process bounding the original system's dynamics. We build upon previous work on multi-agent systems with switching communication networks. Though conservative, our approach provide conditions that can be verified a priori. Our result is illustrated through simulations.
\end{abstract}

\section{INTRODUCTION}

Cooperative behaviors generating complex phenomena are observed in nature [1], [2]. Multi-agent systems also find applications in technical areas such as mobile sensor networks [3], cooperative robotics [4] or distributed implementation of algorithms [5]. A central question arising in the study of multi-agent systems is whether the group will be able to reach a consensus. Intuitively, agents are said to reach a consensus when all individuals agree on a common value (e.g. the heading direction of a flock of birds, the candidate to elect for voters).

To carry out formal studies on consensus problems, one usually assumes that the multi-agent system follows some abstract communication protocol and then investigates conditions under which a consensus will be reached. Existing frameworks include discrete and continuous-time systems involving or neglecting time-delays in the communication process. The communication network between agents is usually modeled by a graph. Its topology is either assumed to be fixed or can switch over time. The switching topology of the interactions is sometimes assumed to depend on the state of the agents (e.g. the strength of the communication can be a function of the distance between agents).

Consensus can be modeled in a deterministic fashion, however, in many applications it seems that the topology of the network is quite random. Recently there has been a growing interest in studying consensus algorithms in a probabilistic setting [6], [7], [8], [9], [10], [11], [12], where network changes can be independent, identically distributed (i.i.d. ) over time [9], ergodic-stationary [13], or Markovian [14].

This research has been partially supported by the ANR (project VEDECY).

S. Martin and A. Girard are with the Laboratoire Jean Kuntzmann, Université de Grenoble, B.P. 53, 38041 Grenoble Cedex 9, France. Samuel.Martineimag.fr. A. Fazeli and A. Jadbabaie are with the Department of Electrical and Systems Engineering at University of Pennsylvania.
This randomness can be due to the unpredictability of the environment in which the communication between agents occurs or due to the inherent probabilistic characteristic of the communication among agents [15].

An example where stochastic consensus protocols apply is when agents in the network communicate if they are within some distance which is governed by some random process. For instance in wireless networks, the log-normal shadowing statistical model can be approximated assuming that the range within which agents communicate is random and uniformly distributed over some interval [16]. Therefore, in these probabilistic cases one should investigate conditions under which consensus will be reached with some given probability. It would be quite useful if a lower bound for this probability could be found as well.

Most papers have investigated sufficient conditions ensuring asymptotic consensus. The assumptions made in the models are usually rather general (see e.g. [17]). This enables the given conditions to apply in a wide range of cases. Conditions usually require some connectivity properties on the expected communication network to hold over time. A drawback in such conditions is that they often cannot be verified a priori. In a random setting, however, sufficient conditions could only ensure asymptotic consensus in probability.

In this paper we consider a group of agents with second order dynamics. We extend previous work from Martin and Girard [18] by considering the following stochastic setting : The communication network is determined by a standard interaction rule based on the distance between agents. The distance within which agents communicate is bounded by some random radius which is i.i.d. over time. The goal of this paper is to determine practical conditions (on the initial positions and velocities of agents) ensuring that the agents eventually agree on a common velocity (i.e. a flocking behavior is achieved) with some probability. These conditions yield a bound on this probability of flocking which depends on the initial configuration only. As defined, the stochastic dynamics is state dependent and therefore not i.i.d. . We exhibit an i.i.d. bounding process which allows us to build upon previous work such as [19], to establish such conditions. Though conservative, our approach provides conditions that can be verified a priori. Moreover, it is computationally tractable and can be fully automated. Our result is illustrated through simulations.

Related results in the literature include [20] where the authors assume a hierarchy in the communication network and [21] where the authors consider additive noise to the dynamics (which makes the asymptotic velocity alignment 
impossible to achieve).

\section{Problem Formulation}

In this paper, we study a discrete time, multi-agent system. We consider a set $\mathcal{V}=\{1, \ldots, n\}$ of mobile agents evolving in a $d$-dimensional space. Each agent $i \in \mathcal{V}$ is characterized by its position $x_{i}(t) \in \mathbb{R}^{d}$ and its velocity $v_{i}(t) \in \mathbb{R}^{d}$. The initial positions and velocities are given by $x_{i}(0)=x_{i}^{0}$ and $v_{i}(0)=v_{i}^{0}$. The agents exchange information over a communication network. The topology of the network depends on the relative position of agents and is therefore subject to change. The agents use the available information to adapt their velocity in order to achieve a flocking behavior. Formally, the evolution of each agent $i \in \mathcal{V}$ is described by the following discrete-time protocol :

$$
\begin{aligned}
& x_{i}(t+1)=x_{i}(t)+\varepsilon v_{i}(t) \\
& v_{i}(t+1)=v_{i}(t)+\varepsilon \sum_{j \in \mathcal{V}} a_{i j}(t)\left(v_{j}(t)-v_{i}(t)\right)
\end{aligned}
$$

where $\varepsilon>0$ is a given parameter of the system and $a_{i j}(t)$ is the communication weight between $i$ and $j$. The weight $a_{i j}(t)=1$ when communication between agents $j$ and $i$ takes place at time $t$ and 0 otherwise. In this paper we focus on communication of agents defined by a metric rule parametrized by a random interaction radius as follows :

$$
a_{i j}(t)=\mathbf{1}_{\left\|x_{i}(t)-x_{j}(t)\right\| \leq R_{i j}(t) \text { and } i \neq j}
$$

where 1 is the indicative function, $\|$.$\| denotes the Euclidean$ norm ${ }^{1}$ and $R_{i j}(t)$ is a positive random variable standing for the radius within which agents $i$ and $j$ are able to communicate at time $t$. We assume that $R_{i j}=R_{j i}$ which makes the communication network given by metric interactions symmetric (i.e. if agent $i$ receives information from agent $j, i$ also sends information to $j$ ). In the rest of the paper, we shall assume that these processes are independent and identically distributed (i.i.d. ). Rigorously, we should write $R_{i j}(t, \omega)$ where $\omega \in \Omega$, the set of probabilistic events. For simplicity of notation, we drop $\omega$, unless necessary. Metric interactions are usually assumed to be a good representation of how collective behavior takes place. Thus, most of the literature on the subject, including [22], [23], uses them.

As defined, the system considered is a discrete-time Markov process. For a realization of the processes $R_{i j}$, we say that the agents achieve a flocking behavior if all the agents asymptotically move with a common direction :

$$
\forall i, j \in \mathcal{V}, \lim _{t \rightarrow+\infty}\left|v_{i}(t)-v_{j}(t)\right|=0 .
$$

The goal of this paper is to determine a lower bound on the probability that the flocking behavior is achieved. This bound should be easily verifiable using only the initial positions and velocities of agents.

\section{PRELIMINARIES}

In this section, we review some results from algebraic graph theory and multi-agent systems that will be useful in the subsequent discussion.

1. In the following, $\|$.$\| will denote the usual Euclidean norm on \mathbb{R}^{d}$ or $\mathbb{R}^{\text {nd }}$ depending on the context.

\section{A. Algebraic Graph Theory}

Let us recall some standard results from algebraic graph theory. More details can be found, for instance, in [24], [25].

Denote the communication weight matrix $A(t)=\left[a_{i j}(t)\right]$. We only consider the case where $A$ is symmetric with entries in $\{0,1\}$ according to the definition of the communication weights. For the rest of the section, we drop the dependence on time. Let $D=\operatorname{diag}\left(d_{1}, d_{2}, \ldots, d_{n}\right)$ be the degree matrix of $A$ where $d_{i}=\sum_{j \in \mathcal{V}} a_{i j}$. Let $L=D-A$ be the laplacian matrix of $A$. $L$ is symmetric, positive-semidefinite. Its eigenvalues are non-negative reals and its smallest one is 0 with eigenvector $\mathbf{1}_{n}$, vector of all ones. We denote these eigenvalues as

$$
0=\lambda_{1}(L) \leq \lambda_{2}(L) \leq \ldots \leq \lambda_{n}(L)
$$

The second smallest eigenvalue of $L, \lambda_{2}(L)$ is usually referred to as the algebraic connectivity of the network. Let $\varepsilon>0$. Let $W=I-\varepsilon L$. $W$ is symmetric and therefore also has real eigenvalues. We denote them as follows :

$$
1=\lambda_{1}(W) \geq \lambda_{2}(W) \geq \ldots \geq \lambda_{n}(W) .
$$

Lemma 1 (Lemma 6 [26]): The eigenspectra of matrices $W$ and $L$ verify for $i \in \mathcal{V}$

$$
\lambda_{i}(W)=1-\varepsilon \lambda_{i}(L) .
$$

Lemma 2 (Lemma 8 [26]): Let $J=\frac{1}{n} 1_{n} 1_{n}^{T}$. The spectral radius of $W-J$ is $\max \left(1-\varepsilon \lambda_{2}(L),-1+\varepsilon \lambda_{n}(L)\right)$.

Remark 3: In the rest of the paper, we shall assume $\varepsilon<\frac{1}{\lambda_{n}(L)}$ for all laplacian matrix considered. This is always possible by choosing $\varepsilon=\frac{1}{2(n-1)}$ (see e.g. [24]). Under this assumption, the spectral radius is $1-\varepsilon \lambda_{2}(L)$. This assumption also yields $\varepsilon<\frac{1}{\lambda_{2}(L)}$ and thus $1-\varepsilon \lambda_{2}(L)>0$.

To a communication weight matrix $A$, we associate a graph $G=(\mathcal{V}, \mathcal{E})$ consisting of a set of nodes $\mathcal{V}=$ $\{1, \ldots, n\}$ and a set of edges given by a relation $\mathcal{E} \subseteq \mathcal{V} \times \mathcal{V}$ such that $(i, j) \in \mathcal{E}$ iff $a_{i j}=1$. $\mathcal{E}$ is symmetric $((i, j) \in \mathcal{E}$ iff $(j, i) \in \mathcal{E})$ and anti-reflexive $(\forall i \in \mathcal{V},(i, i) \notin \mathcal{E})$. If $(i, j) \in \mathcal{E}$, we say that $i$ is a neighbor of $j$. A path between $i$ and $j$ is a sequence of nodes $\left(i_{1}, i_{2}, \ldots, i_{p}\right)$ such that $i_{1}=i, i_{p}=j$ and $\forall k \in\{1, \ldots, p-1\},\left(i_{k}, i_{k+1}\right) \in \mathcal{E}$. We shall consider throughout this paper paths without loops, i.e. for all $k, k^{\prime} \in\{1, \ldots, p-1\} k \neq k^{\prime}$ implies $i_{k} \neq i_{k^{\prime}}$. A graph is said to be connected if for every couple of nodes $(i, j) \in \mathcal{V} \times \mathcal{V}$ such that $i \neq j$, there exists a path between $i$ and $j$. A graph $G^{\prime}=\left(\mathcal{V}, \mathcal{E}^{\prime}\right)$ is said to be a (spanning) subgraph of $G$ if $\mathcal{E}^{\prime} \subseteq \mathcal{E}$.

The following proposition shows that $\lambda_{2}(L)$ is a good measure of the connectivity of the graph :

Lemma 4 ([24]): Let $L$ and $L^{\prime}$ be the laplacians matrices associated to graphs $G$ and $G^{\prime}$ respectively.

- $G$ is connected iff $\lambda_{2}(L)>0$.

- If $G^{\prime}$ is a subgraph of $G$ then $\lambda_{2}\left(L^{\prime}\right) \leq \lambda_{2}(L)$. 


\section{B. Consensus over Random Networks}

In this section, we apply the algebraic formalism to system (1) to derive a useful result. This approach was for instance used in [19] and [26].

Let $x(t)=\left(x_{1}(t), \ldots, x_{n}(t)\right) \in \mathbb{R}^{n d}$ and $v(t)=$ $\left(v_{1}(t), \ldots, v_{n}(t)\right) \in \mathbb{R}^{n d}$ be the stacked vectors of positions and velocities, respectively. We also define the stacked vectors of initial positions and velocities : $x^{0}=\left(x_{1}^{0}, \ldots, x_{n}^{0}\right) \in$ $\mathbb{R}^{n d}$ and $v^{0}=\left(v_{1}^{0}, \ldots, v_{n}^{0}\right) \in \mathbb{R}^{n d}$. Let $\mathbf{L}(t)=L(t) \otimes I_{d}$, $\mathbf{W}(t)=W(t) \otimes I_{d}$ and $\mathbf{J}(t)=J(t) \otimes I_{d}$ where $I_{d}$ is the $d \times d$ identity matrix and $\otimes$ denotes the Kronecker product. Then, system (1) becomes in matrix form

$$
\begin{aligned}
& x(t+1)=x(t)+\varepsilon v(t) \\
& v(t+1)=\mathbf{W}(t) v(t)=\left(I_{n d}-\varepsilon \mathbf{L}(t)\right) v(t) .
\end{aligned}
$$

Since $L(t)$ is symmetric and $\mathbf{1}_{n}$ is an eigenvector associated to eigenvalue 0 , the average of the velocities is preserved by (3). It follows that if the agents achieve a flocking behavior, the common asymptotic velocity is necessarily $v^{*}$.

For $i \in \mathcal{V}$, let $\delta_{i}(t)=v_{i}(t)-v^{*}$, we define the velocity disagreement vector $\delta(t)=\left(\delta_{1}(t), \ldots, \delta_{n}(t)\right)$. Let $y_{i}(t)=$ $x_{i}(t)-\varepsilon v^{*} t$ and $y(t)=\left(y_{1}(t), \ldots, y_{n}(t)\right)$. Denote $z(t)=$ $y(t)-x^{0}$. We have $z(0)=0$. The vector $y(t)$ essentially gives the relative positions of the agents, as we have

$$
\forall i, j \in \mathcal{V}, x_{i}(t)-x_{j}(t)=y_{i}(t)-y_{j}(t)
$$

whereas $z(t)$ gives the modifications of the relative positions since

$$
\forall i, j \in \mathcal{V}, z_{i}(t)-z_{j}(t)=x_{i}(t)-x_{j}(t)-\left(x_{i}^{0}-x_{j}^{0}\right) .
$$

The dynamics of $z$ and $\delta$ are as follows :

$$
\begin{aligned}
& z(t+1)=z(t)+\varepsilon \delta(t), \\
& \delta(t+1)=(\mathbf{W}(t)-\mathbf{J}) \delta(t) .
\end{aligned}
$$

Lemma 5: For all time $t \in \mathbb{N}$,

$$
\|\delta(t+1)\| \leq\left(1-\varepsilon \lambda_{2}(L(t))\right)\|\delta(t)\|
$$

Proof: Using the symmetry of $\mathbf{W}(t)-\mathbf{I}$, we have

$\|\delta(t+1)\|^{2}=\delta(t)^{T}(\mathbf{W}(t)-\mathbf{I})^{2} \delta(t) \leq(\mu(\mathbf{W}(t)-\mathbf{I}))^{2}\|\delta(t)\|^{2}$,

where $\mu(\cdot)$ is the spectral radius. Since the Kronecker product with $I_{d}$ preserves the spectrum, Remark 3 allows us to prove the Lemma.

Lemma 5 gives a sufficient condition for flocking, i.e. $\prod_{t=0}^{\infty}\left(1-\varepsilon \lambda_{2}(L(t))\right)=0$. However, this condition is not simple to verify since $L(t)$ is a position dependent random matrix. This is why, in the next section, we introduce a bounding function of $\|\delta(t)\|$ which is easier to deal with.

\section{PROBABILITY OF FLOCKING}

\section{A. Main result}

Finding sufficient conditions for flocking requires the choice of arbitrary bounds on the initial distances amongt agents : For $i, j \in V$, define $r_{i j}=r_{j i} \in \mathbb{R}^{+} \cup\{+\infty\}$ satisfying

$$
\left\|x_{i}(0)-x_{j}(0)\right\| \leq r_{i j}
$$

We discuss the optimal way to choose such bounds in section $\mathrm{V}$-A. We define weights

$$
\tilde{a}_{i j}(t)=\mathbf{1}_{r_{i j} \leq R_{i j}(t)},
$$

matrix $\tilde{A}(t)=\left[\tilde{a}_{i j}(t)\right], \tilde{G}(t)$ and $\tilde{L}(t)$ the graph and laplacian associated to matrix $\tilde{A}(t)$. Since $R_{i j}(t)$ are i.i.d. processes, so are $\tilde{a}_{i j}(t), \tilde{A}(t)$ and $\tilde{L}(t)$. Thus, $\mathbb{E}\left(\lambda_{2}(\tilde{L}(t))\right)$ is independent of time. Then, let $\tilde{\lambda}_{2}=\mathbb{E}\left(\lambda_{2}(\tilde{L}(t))\right)$. In the rest of the paper, we will assume $\tilde{\lambda}_{2}>0$. This occurs iff $\tilde{G}(t)$ is connected with non-zero probability $\left(e . g . r_{i j}=\right.$ $\left\|x_{i}(0)-x_{j}(0)\right\|$ for $i \neq j$ is a suitable choice given that $G(0)$ is connected with non-zero probability). Notice that, as defined, $\tilde{G}(t)$ is a subgraph of $G(0)$. Thus, according to Lemma $4, \lambda_{2}(L(0)) \geq \lambda_{2}(\tilde{L}(0))$. Then, if the bound given by Lemma 5 is tight, the communication according to topology $G(0)$ will yield a faster velocity alignment than $\tilde{G}(0)$. If we can show that this remains true over time (see Lemma 8), flocking is more likely to occur in the former than in the later case. Bounding the flocking probability of the group evolving according to $\tilde{G}$ will allow us to conclude. We formalize this discussion in Section IV-B. The $r_{i j}$ define upper bounds on the initial distances between agents. Using $r_{i j}$, we define $\rho$, the robustness of these bounds with regards to the agents' distances (i.e. the allowed perturbation on the positions before reaching one of these bounds) as follows :

$$
\rho=\min _{i, j \in \mathcal{V}} r_{i j}-\left\|x_{i}(0)-x_{j}(0)\right\| .
$$

The previous definitons suffice to state the main result of the paper. This should help to see the objective of the derivations to come.

Theorem 6: Assume that $\rho>0$ and $\tilde{\lambda}_{2}>0$. Moreover suppose $\|\delta(0)\| \leq \frac{\rho \tilde{\lambda}_{2}}{\sqrt{2}}$. Then the probability that flocking is achieved is higher than

$$
1-\frac{1}{1+\kappa^{2}} \text { where } \kappa=\frac{1}{\nu_{\varepsilon}}\left(\frac{\rho \tilde{\lambda}_{2}}{\sqrt{2}\|\delta(0)\|}-1\right)^{2}
$$

and $\nu_{\varepsilon}=\frac{\varepsilon\left(\mathbb{E}\left(\lambda_{2}(\tilde{L}(t))^{2}\right)-\tilde{\lambda}_{2}^{2}\right)}{\left(2 \tilde{\lambda}_{2}-\varepsilon \mathbb{E}\left(\lambda_{2}(\tilde{L}(t))^{2}\right)\right)} \in[0,1]$.

Remark 7: As given, the result allows us to obtain the probability of flocking arbitrarily close to 1 in two ways. First, one can choose the initial disagreement vector sufficiently small. Second, since $\lim _{\varepsilon \rightarrow 0} \nu_{\varepsilon}=0$, assume the condition on $\|\delta(0)\|$ is satisfied, and then choose $\varepsilon$ sufficiently small.

The proof of the theorem requires the introduction of a bounding process and is therefore relocated at the end of the next section.

\section{B. Bounding process}

We give a system of two real variables $(p, q)$ which will play the role of bounding process for the multi-agent system :

$$
\begin{aligned}
& p(t+1)=p(t)+\varepsilon q(t) \\
& q(t+1)=\left(1-\varepsilon \lambda_{2}(\tilde{L}(t))\right) q(t)
\end{aligned}
$$

with $p(0)=0$ and $q(0)=\|\delta(0)\|$. Since $\lambda_{2}(\tilde{L}(t))$ is i.i.d. , the behavior of $(p, q)$ will be easy to characterize. Under certain conditions, we shall show that $p$ bounds $\|z\|$, 
the distance modification between agents' positions while $q$ serves as a bound for $\|\delta\|$ the distance to velocity alignment (see equations (8) and (5)).

The definition of the robustness $\rho$ allows to state the following lemma :

Lemma 8: Let $z(t)=y(t)-x^{0}$ for $t \geq 0$ as in Section IIIB. For all $t \in \mathbb{N}$, if

$$
\|z(t)\| \leq \frac{\rho}{\sqrt{2}}
$$

then $\tilde{G}(t)$ is a subgraph of $G(t)$.

Proof: Let $i, j \in \mathcal{V}$. Using equation (4), we have

$$
\begin{aligned}
& \left\|x_{i}(t)-x_{j}(t)\right\|=\left\|x_{i}(t)-x_{j}(t)-\left(x_{i}^{0}-x_{j}^{0}\right)+\left(x_{i}^{0}-x_{j}^{0}\right)\right\| \\
& \leq\left\|x_{i}(t)-x_{j}(t)-\left(x_{i}^{0}-x_{j}^{0}\right)\right\|+\left\|x_{i}^{0}-x_{j}^{0}\right\| \\
& \leq\left\|z_{i}(t)-z_{j}(t)\right\|+\left\|x_{i}^{0}-x_{j}^{0}\right\| \\
& \leq \sqrt{2}\|z(t)\|+\left\|x_{i}^{0}-x_{j}^{0}\right\| \leq \rho+\left\|x_{i}^{0}-x_{j}^{0}\right\| \leq r_{i j} .
\end{aligned}
$$

This shows that

$$
a_{i j}(t)=\mathbf{1}_{\left\|x_{i}(t)-x_{j}(t)\right\| \leq R_{i j}(t)} \geq \mathbf{1}_{r_{i j} \leq R_{i j}(t)}=\tilde{a}_{i j}(t)
$$

which concludes the proof.

We can now state the bounding property :

Lemma 9: We have

$$
\left(\forall t \in \mathbb{N}, p(t)<\frac{\rho}{\sqrt{2}}\right) \Rightarrow\left(\forall t \in \mathbb{N},\left\{\begin{array}{l}
\|\delta(t)\| \leq q(t) \\
\|z(t)\| \leq p(t)
\end{array}\right) .\right.
$$

Proof: We show the result by induction on time. Assume that the left-hand side of the implication is satisfied. By definition, $q(0)=\|\delta(0)\|$ and $p(0)=\|z(0)\|=0$. Assume the result is true for some time $t:\|\delta(t)\| \leq q(t)$ and $\|z(t)\| \leq p(t)$. The inequality on the positions at time $t+1$ comes as follows :

$$
\begin{aligned}
\|z(t+1)\| & =\|z(t)+\varepsilon \delta(t)\| \leq\|z(t)\|+\varepsilon\|\delta(t)\| \\
& \leq p(t)+\varepsilon q(t)=p(t+1) .
\end{aligned}
$$

For the inequality on the velocities, we have $\|z(t)\| \leq p(t)<$ $\rho / \sqrt{2}$, where we used the left-hand side of the implication. Thus, we can apply Lemma 8 along with Lemma 4 to obtain $\lambda_{2}(L(t)) \geq \lambda_{2}(\tilde{L}(t))$. Then, Lemma 5 gives

$$
\begin{aligned}
\|\delta(t+1)\| & \leq\left(1-\varepsilon \lambda_{2}(L(t))\right)\|\delta(t)\| \\
& \leq\left(1-\varepsilon \lambda_{2}(\tilde{L}(t))\right)\|\delta(t)\| \\
& \leq\left(1-\varepsilon \lambda_{2}(\tilde{L}(t))\right) q(t)=q(t+1) .
\end{aligned}
$$

This last lemma leads to the following intermediate result :

Theorem 10: Consider a given realization of processes $R_{i j}$. If for all time $t, p(t) \leq \frac{\rho}{\sqrt{2}}$ then flocking is achieved, i.e. $\lim _{t \rightarrow+\infty}\|\delta(t)\|=0$.

Proof: $q$ is non-negative and non-increasing, thus it must converge. It is easy to see that if $\lim _{t \rightarrow \infty} q(t)>0$ then $p$ diverges. Thus, $\lim _{t \rightarrow \infty} q(t)=0$. Using Lemma 9, we have $\|\delta(t)\| \leq q(t)$, thus $\lim _{t \rightarrow \infty}\|\delta(t)\|=0$ which guarantees flocking.

In order to use the previous Lemma, we need to be able to characterize the evolution of $p$ in function of the initial configurations of the system. This is done in the rest of the section.

Lemma 11: For all $t \in \mathbb{N}$, we have

$$
\left\{\begin{array}{l}
\mathbb{E}(q(t+1))=\alpha \mathbb{E}(q(t)) \\
\mathbb{E}\left(q(t+1)^{2}\right)=\beta \mathbb{E}\left(q(t)^{2}\right)
\end{array}\right.
$$

where

$$
\left\{\begin{aligned}
\alpha & =\mathbb{E}\left(1-\varepsilon \lambda_{2}(\tilde{L}(t))\right)=1-\varepsilon \tilde{\lambda}_{2} \\
\beta & =\mathbb{E}\left(\left(1-\varepsilon \lambda_{2}(\tilde{L}(t))\right)^{2}\right) \\
& =1-2 \varepsilon \tilde{\lambda}_{2}+\varepsilon^{2} \mathbb{E}\left(\lambda_{2}(\tilde{L}(t))^{2}\right) .
\end{aligned}\right.
$$
we have

Proof: By definition, $\tilde{L}(t)$ is independent of $q(t)$, so

$$
\begin{aligned}
\mathbb{E}(q(t+1)) & =\mathbb{E}\left(\left(1-\varepsilon \lambda_{2}(\tilde{L}(t))\right) q(t)\right) \\
& =\left(1-\varepsilon \tilde{\lambda}_{2}\right) \mathbb{E}(q(t))=\alpha \mathbb{E}(q(t))
\end{aligned}
$$

Similarly,

$$
\mathbb{E}\left(q(t+1)^{2}\right)=\mathbb{E}\left(\left(1-\varepsilon \lambda_{2}(\tilde{L}(t))\right)^{2} q(t)^{2}\right)=\beta \mathbb{E}\left(q(t)^{2}\right)
$$

The bounding process is useful only if the variance of $q(t)$ converges to 0 . This is the case under the condition assumed in Remark 3 regarding $\varepsilon$ :

Remark 12: Assuming $\varepsilon<1 / \lambda_{n}(\tilde{L}(t))$ for all $t$ as in Remark 3, both $\alpha$ and $\beta$ lie in interval ]0,1[.

The previous remark holds since, according to Remark 3, $\left.1-\varepsilon \lambda_{2}(\tilde{L}(t)) \in\right] 0,1[$ for any realization of the random radius $R(t)$ and any choice $r_{i j}$. Then, the definition of $\alpha$ and $\beta$ allows us to conclude.

A direct corollary of Lemma 11 is the following :

Corollary 13: For all $t, u \in \mathbb{N}$, we have

$$
\left\{\begin{array}{l}
\mathbb{E}(q(t))=\alpha^{t} q(0) \\
\mathbb{E}\left(q(t)^{2}\right)=\beta^{t} q(0)^{2} \\
\mathbb{E}(q(t+u) q(t))=\alpha^{u} \mathbb{E}\left(q(t)^{2}\right)
\end{array}\right.
$$

Proof: The first and second equations are straightforward. The third one is also a consequence of $\tilde{L}(t)$ being i.i.d. :

$$
\begin{aligned}
\mathbb{E}(q(t+u) q(t)) & =\mathbb{E}\left(\prod_{j=0}^{u-1}\left(1-\varepsilon \lambda_{2}(\tilde{L}(t+j))\right) q(t)^{2}\right) \\
& =\left(1-\varepsilon \mathbb{E}\left(\lambda_{2}(\tilde{L}(t))\right)^{u} \mathbb{E}\left(q(t)^{2}\right)\right.
\end{aligned}
$$

Noticing that $p(t)=\varepsilon \sum_{k=0}^{t-1} q(k)$ and with the assumption $\tilde{\lambda}_{2}>0$, the following also holds :

Lemma 14: If we assume $\tilde{\lambda}_{2}>0$, we have, for all $t \in \mathbb{N}$,

$$
\left\{\begin{array}{l}
\mathbb{E}(p(t))=\varepsilon \frac{q(0)}{1-\alpha}+o(t) \\
\sigma^{2}(p(t))=\varepsilon^{2} \frac{\left(\beta-\alpha^{2}\right) q(0)^{2}}{(1-\alpha)^{2}(1-\beta)}+o(t)
\end{array}\right.
$$

where $\sigma^{2}(p(t))=\mathbb{E}\left(p(t)^{2}\right)-\mathbb{E}(p(t))^{2}$ and $o(t)$ is a function converging to 0 when time $t$ approaches to infinity. 
Proof: The first equality comes from the above remark and the first equation in Corollary 13 :

$$
\begin{aligned}
\mathbb{E}(p(t)) & =\varepsilon \sum_{k=0}^{t-1} \mathbb{E}(q(k))=\varepsilon \sum_{k=0}^{t-1} \alpha^{k} q(0) \\
& =\varepsilon \frac{1-\alpha^{t}}{1-\alpha} q(0)=\varepsilon \frac{q(0)}{1-\alpha}+o(t)
\end{aligned}
$$

where we used $|\alpha|<1$ as given in Remark 12 .

For the second equality,

$$
\begin{aligned}
p(t)^{2} & =\varepsilon^{2} \sum_{k, j=0}^{t-1} q(k) q(j) \\
& =\varepsilon^{2}\left(\sum_{k=0}^{t-1} q(k)^{2}+2 \sum_{k<j} q(j) q(k)\right) \\
& =\varepsilon^{2} \sum_{k=0}^{t-1}\left(q(k)^{2}+2 \sum_{u=1}^{t-1-k} q(k+u) q(k)\right) .
\end{aligned}
$$

Using the linearity of the expectation, Corollary 13 and $|\alpha|<$ 1 , we obtain

$$
\begin{aligned}
\mathbb{E}\left(p(t)^{2}\right) & =\varepsilon^{2} \sum_{k=0}^{t-1}\left(\mathbb{E}\left(q(k)^{2}\right)+2 \sum_{u=1}^{t-1-k} \alpha^{u} \mathbb{E}\left(q(k)^{2}\right)\right) \\
& =\varepsilon^{2} \sum_{k=0}^{t-1}\left(1+2\left(\frac{1-\alpha^{t-k}}{1-\alpha}-1\right)\right) \mathbb{E}\left(q(k)^{2}\right) \\
& =\varepsilon^{2} \sum_{k=0}^{t-1}\left(\frac{1+\alpha}{1-\alpha}-\left(\frac{2 \alpha^{t}}{1-\alpha} \alpha^{-k}\right)\right) \beta^{k} q(0)^{2} \\
& =\varepsilon^{2}\left(\frac{1+\alpha}{1-\alpha} \sum_{k=0}^{t-1} \beta^{k}-\frac{2 \alpha^{t}}{1-\alpha} \sum_{k=0}^{t-1}\left(\frac{\beta}{\alpha}\right)^{k}\right) q(0)^{2} .
\end{aligned}
$$

Moreover, we have

$$
\beta=1-2 \varepsilon \tilde{\lambda}_{2}+\varepsilon^{2} \mathbb{E}\left(\lambda_{2}(\tilde{L}(t))^{2}\right)<1-2 \varepsilon \tilde{\lambda}_{2}+\varepsilon \tilde{\lambda}_{2}=\alpha .
$$

Thus, $\beta \neq \alpha$. Also $|\beta|<1$, so we have

$$
\begin{aligned}
& \mathbb{E}\left(p(t)^{2}\right)=\varepsilon^{2}\left(\frac{1+\alpha}{1-\alpha} \frac{1-\beta^{t}}{1-\beta}-\frac{2 \alpha^{t}}{1-\alpha} \frac{1-(\beta / \alpha)^{t}}{1-(\beta / \alpha)}\right) q(0)^{2} \\
& =\varepsilon^{2}\left(\frac{1+\alpha}{1-\alpha} \frac{1-\beta^{t}}{1-\beta}-\frac{2}{1-\alpha} \frac{\alpha^{t}-\beta^{t}}{1-(\beta / \alpha)}\right) q(0)^{2} \\
& =\varepsilon^{2} \frac{1+\alpha}{(1-\alpha)(1-\beta)} q(0)^{2}+o(t) .
\end{aligned}
$$

Then,

$$
\begin{aligned}
& \sigma^{2}(p(t))=\mathbb{E}\left(p(t)^{2}\right)-\mathbb{E}(p(t))^{2} \\
& =\varepsilon^{2}\left(\frac{1+\alpha}{(1-\alpha)(1-\beta)}-\frac{1}{(1-\alpha)^{2}}\right) q(0)^{2}+o(t) .
\end{aligned}
$$

Reformulating the last right-hand side leads to the expected result.

Finally, we translate the equation from the previous lemma in terms of the expectation and deviation of the algebraic connectivity :
Corollary 15: For all $t \in \mathbb{N}$, we have

$$
\left\{\begin{array}{l}
\mathbb{E}(p(t))=\frac{\|\delta(0)\|}{\tilde{\lambda}_{2}}+o(t), \\
\sigma^{2}(p(t))=\frac{\nu_{\varepsilon}\|\delta(0)\|^{2}}{\tilde{\lambda}_{2}^{2}}+o(t),
\end{array}\right.
$$

where $\nu_{\varepsilon} \in[0,1]$ is defined in Theorem 6 .

Remark 16: Since $\lim _{\varepsilon \rightarrow 0} \nu_{\varepsilon}=0$, the previous result shows that one can obtain the limit variance of $p(t)$ as small as desired by choosing $\varepsilon$ small enough.

Proof: Notice that the definitions of $\alpha$ and $\beta$ give

$$
\begin{aligned}
& 1-\alpha=\varepsilon \tilde{\lambda}_{2}, 1-\beta=\varepsilon\left(2 \tilde{\lambda}_{2}-\varepsilon \mathbb{E}\left(\lambda_{2}(\tilde{L}(t))^{2}\right)\right), \\
& 1+\alpha=2-\varepsilon \tilde{\lambda}_{2}, \beta-\alpha^{2}=\varepsilon^{2}\left(\mathbb{E}\left(\lambda_{2}(\tilde{L}(t))^{2}\right)-\tilde{\lambda}_{2}^{2}\right) .
\end{aligned}
$$

Then, the first equation is straightforward. The second is derived as follows :

$$
\begin{aligned}
\sigma^{2}(p(t)) & =\varepsilon^{2} \frac{\left(\beta-\alpha^{2}\right) q(0)^{2}}{(1-\alpha)^{2}(1-\beta)}+o(t) \\
& =\varepsilon^{4} \frac{\left(\mathbb{E}\left(\lambda_{2}(\tilde{L}(t))^{2}\right)-\tilde{\lambda}_{2}^{2}\right)\|\delta(0)\|^{2}}{\varepsilon^{3} \tilde{\lambda}_{2}^{2}\left(2 \tilde{\lambda}_{2}-\varepsilon \mathbb{E}\left(\lambda_{2}(\tilde{L}(t))^{2}\right)\right)}+o(t) \\
& =\frac{\nu_{\varepsilon}\|\delta(0)\|^{2}}{\tilde{\lambda}_{2}^{2}}+o(t) .
\end{aligned}
$$

The fact that $\nu_{\varepsilon} \geq 0$ can be deduced from $\sigma^{2}(p(t) \geq 0$. The inequality $\nu_{\varepsilon} \leq 1$ comes from Remark $3: 0<\varepsilon<\frac{1}{\lambda_{2}(\tilde{L}(t))}$ for any realization of the random radius $R(t)$ and choice $r_{i j}$. Thus, $\varepsilon \lambda_{2}(\tilde{L}(t))^{2}<\lambda_{2}(\tilde{L}(t))$ which gives

$$
\varepsilon \mathbb{E}\left(\lambda_{2}(\tilde{L}(t))^{2}\right)=\mathbb{E}\left(\varepsilon \lambda_{2}(\tilde{L}(t))^{2}\right)<\mathbb{E}\left(\lambda_{2}(\tilde{L}(t))\right)=\tilde{\lambda}_{2} .
$$

Thus, $\tilde{\lambda}_{2}<2 \tilde{\lambda}_{2}-\varepsilon \mathbb{E}\left(\lambda_{2}(\tilde{L}(t))^{2}\right)$ and

$$
\nu_{\varepsilon}<\varepsilon \frac{\left(\mathbb{E}\left(\lambda_{2}(\tilde{L}(t))^{2}\right)-\tilde{\lambda}_{2}^{2}\right)}{\tilde{\lambda}_{2}} \leq \varepsilon \frac{\mathbb{E}\left(\lambda_{2}(\tilde{L}(t))^{2}\right)}{\tilde{\lambda}_{2}}<1 .
$$

We can now give the proof of the central theorem of this paper.

Proof: [Proof of Theorem 6] Theorem 10 shows the probability that flocking occurs is higher than the probability that for all time $t, p(t) \leq \frac{\rho}{\sqrt{2}}$. Denote $\mathcal{A}_{t}=\{\omega \in \Omega \mid p(t) \leq$ $\left.\frac{\rho}{\sqrt{2}}\right\}$. Since $p$ is increasing, $\left(\mathcal{A}_{t}\right)$ is a decreasing sequence of events and we have

$$
\begin{aligned}
& \mathbb{P}\left(\forall t, p(t) \leq \frac{\rho}{\sqrt{2}}\right)=\mathbb{P}\left(\cap_{t \geq 0} \mathcal{A}_{t}\right) \\
& =\lim _{t \rightarrow+\infty} \mathbb{P}\left(\mathcal{A}_{t}\right)=\lim _{t \rightarrow+\infty} \mathbb{P}\left(p(t) \leq \frac{\rho}{\sqrt{2}}\right) \\
& =1-\lim _{t \rightarrow+\infty} \mathbb{P}\left(p(t)>\frac{\rho}{\sqrt{2}}\right) .
\end{aligned}
$$

The bound on the probability of flocking comes from the one sided Chebyshev's inequality :

$$
\mathbb{P}\left(p(t)>\frac{\rho}{\sqrt{2}}\right) \leq \frac{1}{1+k(t)}
$$

where $k(t)=\frac{\left(\frac{\rho}{\sqrt{2}}-\mathbb{E}(p(t))\right)^{2}}{\sigma^{2}(p(t))}$. We are only interested in the limit when $t$ diverges to infinity. We use Corollary 15 to 
derive a lower bound on the limit of $k(t)$ :

$$
\frac{\left(\frac{\rho}{\sqrt{2}}-\lim _{t \rightarrow \infty} \mathbb{E}(p(t))\right)^{2}}{\lim _{t \rightarrow \infty} \sigma^{2}(p(t))} \geq \frac{1}{\nu_{\varepsilon}}\left(\frac{\rho \tilde{\lambda}_{2}}{\sqrt{2}\|\delta(0)\|}-1\right)^{2} .
$$

\section{APPLICATION TO WIRELESS NETWORKS}

Until now, we have not assumed any constraint on the communication radiuses $R_{i j}(t)$ besides being non-negative i.i.d. random variables satisfying the constraint $R_{i j}(t)=$ $R_{j i}(t)$. In the present section, we apply our result to the case where $R_{i j}(t)=\min \left(R_{i}(t), R_{j}(t)\right)$ where $R_{i}(t)$ follows a uniform distribution over the interval $\left[0, R_{\max }\right]$ where $R_{\max }>0$ is some maximum communication range. $R_{i}(t)$ represents the sensing radius of agent $i$ at time $t$. Taking $R_{i j}(t)=\min \left(R_{i}(t), R_{j}(t)\right)$ means that two agents interact if and only if both agents can sense the other. It has been shown in [16] that the uniform distribution is a good approximation of the log-normal shadowing statistical model for wireless networks and is, as such, of practical relevance.

\section{A. Choice of $r_{i j}$}

The choice of $r_{i j}$ is important since it determines both $\lambda_{2}(\tilde{L}(t))$ (equation (6)) and $\rho$ (equation (7)). Precisely, $\rho$ increases with $r_{i j}$ starting at 0 when $r_{i j}=\left\|x_{i}^{0}-x_{j}^{0}\right\|$ for all $i, j \in \mathcal{V}$ whereas $\lambda_{2}(\tilde{L}(t))$ decreases when $r_{i j}$ increases. According to Theorem 6 , the best bound is achieved when the product $\rho \tilde{\lambda}_{2}$ is highest. The next lemma shows that $\lambda_{2}(\tilde{L}(t))$ can be expressed in term of the robustness $\rho$ only. The idea is to use the fact that $\tilde{G}$ increases when $r_{i j}$ and, as a result, $\rho$ decrease, so that the highest $\lambda_{2}(L)$ is found for the smallest $r_{i j}$ values and, as a result, for smallest $\rho$ :

Lemma 17: Assume that $\rho$ is fixed. Then the choice of $r_{i j}$ maximizing product $\rho \lambda_{2}(\tilde{L}(t))$ is

$$
r_{i j}^{*} \triangleq \rho+\left\|x_{i}^{0}-x_{j}^{0}\right\| .
$$

Proof: Choose some $r_{i j}$ satisfying $\rho=\min _{i, j \in \mathcal{V}} r_{i j}-$ $\left\|x_{i}^{0}-x_{j}^{0}\right\|$. According to the definition, the robustness associated with $r_{i j}^{*}$ is also $\rho: \rho=\min _{i, j \in \mathcal{V}} r_{i j}^{*}-\left\|x_{i}^{0}-x_{j}^{0}\right\|$. Then, denote $\lambda_{2}^{*}(t)$ the algebraic connectivity corresponding to the choice $r_{i j}^{*}$. Since both choices lead to the same robustness, it remains to show that the later choice leads to a higher algebraic connectivity. Let $i, j$ in $\mathcal{V}$ and $t \in \mathbb{N}$. Then, we have

$\tilde{a}_{i j}(t)=\mathbf{1}_{r_{i j} \leq R_{i j}(t)} \leq \mathbf{1}_{\rho+\left\|x_{i}^{0}-x_{j}^{0}\right\| \leq R_{i j}(t)}=\mathbf{1}_{r_{i j}^{*} \leq R_{i j}(t)}=a_{i j}^{*}(t)$.

Thus, $\tilde{G}(t)$ is a subgraph of $G^{*}(t)$. Using Lemma 4 , we obtain $\lambda_{2}(\tilde{L}(t)) \leq \lambda_{2}^{*}(t)$

From now on, we shall assume that the vicinity $r_{i j}$ is chosen as given in Lemma 17, thus we drop the * notation. Since $r_{i j}$ is now a function of $\rho$, so is $\tilde{\lambda}_{2}$. One can show that $\tilde{\lambda}_{2}$ is a polynomial function of $\rho$. We do not explicit this fact because of space limitation.

\section{B. Simulations}

In this section we use a simple 6-agent system to illustrate our theoretical result. Agents initially divide in two groups with velocities bearing opposite $y$-coordinates. The initial positions induce a connected communication network with positive probability. We wonder with which probability the group will overcome the initial conflict and eventually stay together achieving a flocking behavior. According to Theorem 6 , our bound on the probability of flocking depends on the ratio $\frac{\tilde{\lambda}_{2} \rho}{\|\delta(0)\|}$. The numerator is completely determined by the agents' positions (vector $x^{0}$ ) while the denominator is a function of the initial velocities (vector $v^{0}$ ). In order to explore the different outcomes of the system, we fix the initial positions and vary the initial velocities. We set a reference vector of initial velocity disagreement $d=$ $\frac{1}{\sqrt{6}}(1,1,1,-1,-1,-1)^{T}$ so that $\|d\|=1$. We choose the $y$-coordinate of the initial velocity vector $v_{y}^{0}=\gamma d$ where $\gamma$ is the parameter we use to tune the system. We take the $x$-coordinate $v_{x}^{0}=c \mathbf{1}$ where $c>0$ is an arbitrary constant which does not influence the flocking outcome of the dynamics and serves only for visualization purposes. We have $\|\delta(0)\|=\left\|(I-J) v^{0}\right\|=\|\gamma d\|=\gamma$ so $\gamma$ represents the amplitude of the initial velocity disagreement.

Since $x^{0}$ is fixed, we can determine the optimal product $\mathbb{E}\left(\lambda_{2}(\tilde{L})\right) \rho$ for $\rho \in\left[0, R_{\max }\right]$. For the numerical simulation, we use $R_{\max }=15$. The optimization gives $\rho_{\text {opt }}=1.9$ and $\mathbb{E}\left(\lambda_{2}\left(\rho_{\text {opt }}\right)\right) \rho_{\text {opt }}=0.76$. We choose the time step $\varepsilon=0.2$ as suggested in Remark 3 which gives a value $\nu_{\varepsilon}=0.37$.

To illustrate the results, we ran some simulations. First, we chose $\|\delta(0)\|=0.9 \frac{\rho \tilde{\lambda}_{2}}{\sqrt{2}}$ which leads to a lower bound on the probability of flocking of 0.6 . Then, we chose $\|\delta(0)\|=$ $15 \frac{\rho \bar{\lambda}_{2}}{\sqrt{2}}$ for which Theorem 6 does not provide any information regarding the asymptotic behavior. Figure 1 presents snapshots of the evolution of the system for a realization of the random process for the two different choices of $\|\delta(0)\|$ exhibiting different outcome. In the first case, the group asymptotically converges to flocking despite that the graph does not remain connected at all times. The second part of the figure presents a case where the initial velocity disagreement is too high and the two subgroups split apart, never reaching the flocking behavior.

\section{CONCLUSION}

In this paper, we have considered a multi-agent system consisting of mobile agents with second-order dynamics and where the communication network is determined by a metric rule depending on a random interaction range. Our approach extends earlier work from Martin and Girard [18] to the stochastic setting. It links algebraic connectivity of the stochastic communication network to the speed of convergence towards consensus. We have established a lower bound on the probability of velocity alignment depending on the initial positions and velocities of the agents. Our main contribution has been to propose a suitable i.i.d. bounding process to the original system. Our main result states that the probability of flocking can be made higher than any constant arbitrarily 

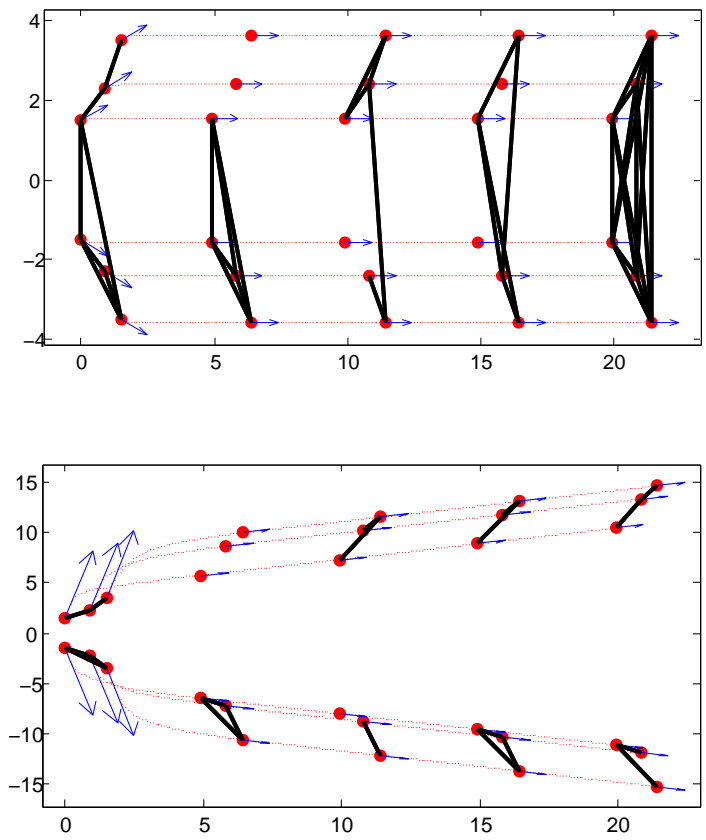

Fig. 1. The upper part of the figure presents an instantiation of the dynamics for $\|\delta(0)\|=0.9 \frac{\rho \tilde{\lambda}_{2}}{\sqrt{2}}$. The lower part is an instantiation when $\|\delta(0)\|=$ $15 \frac{\rho \tilde{\lambda}_{2}}{\sqrt{2}}$. The figure shows the trajectories of the 6 agents and their positions, velocities and connexions for 5 specific times. The six extreme left red dots are the initial agents'positions. The blue arrows represent the velocities (their amplitude has been increased 2 times for visualization purposes). The black lines between dots represents communication link.

close to 1 by choosing a velocity disagreement among agents smaller than a threshold (formed with the robustness and the expected algebraic connectivity of the graph of the bounding process). The main interest of this approach is the possibility of ensuring flocking a priori. The condition can be easily verified through rapid computation.

For future work, we plan to improve the tightness of the bound by taking into account two facts : we will relate velocities with positions because two agents with opposite velocities have more chance to agree on their velocities if they point toward each other, than if they point away from each other. Also, a subgroup of agents with high connectivity is intuitively more inclined to agree on their velocities than a subgroup of low connectivity. Thus, agents belonging to a highly connected local neighborhood should be allowed higher initial velocities.

\section{REFERENCES}

[1] Larissa Conradt and Timothy J. Roper, "Consensus decision making in animals", Trends in Ecology \& Evolution, vol. 20, no. 8, pp. 449-456, 2005.

[2] Iain D. Couzin and Jens Krause, "Self-organization and collective behavior in vertebrates", Advances in the Study of Behavior, vol. Volume 32, pp. 1-75, 2003.

[3] Thomas B. Curtin and James G. Bellingham, "Progress toward autonomous ocean sampling networks", Deep Sea Research Part II : Topical Studies in Oceanography, vol. 56, no. 3-5, pp. 62 - 67, 2009,
AOSN II : The Science and Technology of an Autonomous Ocean Sampling Network.

[4] J. Cortes, S. Martinez, T. Karatas, and F. Bullo, "Coverage control for mobile sensing networks", Robotics and Automation, IEEE Transactions on, vol. 20, no. 2, pp. 243-255, April 2004.

[5] J. N. Tsitsiklis, D. Bertsekas, and M. Athans, "Distributed asynchronous deterministic and stochastic gradient optimization algorithms", IEEE Transactions on Automatic Control, vol. 31, no. 9, pp. 803-812, 1986.

[6] Y. Hatano and M. Mesbahi, "Agreement over random networks", IEEE Transactions on Automatic Control, vol. 50, no. 11, pp. 1867-1872, 2005.

[7] C.W. Wu, "Synchronization and convergence of linear dynamics in random directed networks", IEEE Transactions on Automatic Control, vol. 51, no. 7, pp. 1207-1210, 2006.

[8] M. Porfiri and D.J. Stilwell, "Consensus seeking over random weighted directed graphs", IEEE Transactions on Automatic Control, vol. 52, no. 9, pp. 1767-1773, 2007.

[9] A. Tahbaz-Salehi and A. Jadbabaie, "On consensus over random networks", IEEE Transactions on Automatic Control, vol. 53, no. 3, pp. 791-795, 2008.

[10] G. Picci and T. Taylor, "Almost sure convergence of random gossip algorithms", in IEEE Conf. on Decision and Control, 2007, pp. 282287.

[11] D. Acemoglu, A. Ozdaglar, and A. ParandehGheibi, "Spread of (mis) information in social networks", Games and Economic Behavior, 2010.

[12] B. Touri and A. Nedich, "On ergodicity, infinite flow and consensus in random models", IEEE Transactions on Automatic Control, , no. 99, pp. 1-1, 2010.

[13] A. Tahbaz-Salehi and A. Jadbabaie, "Consensus over ergodic stationary graph processes", IEEE Transactions on Automatic Control, vol. 55, no. 1, pp. 225-230, 2010.

[14] M. Ion and B. John, "Convergence results for the linear consensus problem under Markovian random graphs", Institute for Systems Research Technical Reports, 2009.

[15] F. Fagnani and S. Zampieri, "Randomized consensus algorithms over large scale networks", in Information Theory and Applications Workshop, 2007, 2007, pp. 150-159.

[16] Sergio Bermudez and Stephen B. Wicker, "Connectivity of finite wireless networks with random communication range nodes", in $I C C$ 2009, pp. 1-5.

[17] L. Moreau, "Stability of multiagent systems with time-dependent communication links", IEEE Transactions on Automatic Control, vol. 50, no. 2, pp. 169-182, Feb. 2005.

[18] S. Martin and A. Girard, "Sufficient conditions for flocking via graph robustness analysis", in Decision and Control (CDC), 2010 49th IEEE Conference on, dec. 2010, pp. $6293-6298$.

[19] Alireza Tahbaz-Salehi and Ali Jadbabaie, "On consensus over random networks", in IEEE Conference Decision and Control, 2006.

[20] Federico Dalmao and Ernesto Mordecki, "Cucker-Smale flocking under hierarchical leadership and random interactions.", SIAM J. Appl. Math., vol. 71, no. 4, pp. 1307-1316, 2011.

[21] F. Cucker and E. Mordecki, "Flocking in noisy environments", Journal de Mathématiques Pures et Appliqués, vol. 89, no. 3, pp. 278-296, Mar. 2008.

[22] Craig W. Reynolds, "Flocks, herds, and schools : A distributed behavioral model", Computer Graphics, vol. 21, no. 4, pp. 25-34, 1987.

[23] Tamás Vicsek, András Czirók, Eshel Ben-Jacob, Inon Cohen, and Ofer Shochet, "Novel type of phase transition in a system of self-driven particles", Phys. Rev. Lett., vol. 75, no. 6, pp. 1226-1229, Aug 1995.

[24] Chris Godsil and Gordon Royle, Algebraic Graph Theory, Springer, April 2001.

[25] Russell Merris, "Laplacian matrices of graphs : a survey", Linear Algebra and its Applications, vol. 197-198, pp. 143 - 176, 1994.

[26] Soummya Kar and José M. F. Moura, "Sensor networks with random links : Topology design for distributed consensus", CoRR, vol abs/0704.0954, 2007. 